Tropical Journal of Pharmaceutical Research, June 2007; 6 (2): 695-703

(c) Pharmacotherapy Group, Faculty of Pharmacy, University of Benin Benin City, Nigeria.

All rights reserved.

Research Article

Available online at http://www.tjpr.org

\title{
Improved Colorimetric Determination of Reserpine in Tablets Using 4-Caboxyl-2,6-dintrobenzene diazonium ion (CDNBD)
}

\author{
Olajire A Adegoke, Sunday O Idowu* and Ajibola A Olaniyi
}

Department of Pharmaceutical Chemistry, Faculty of Pharmacy, University of Ibadan, Nigeria

\begin{abstract}
Purpose: To develop a simple, rapid and improved colorimetric method for the assay of reserpine in tablets

Method: The method is based on the aromatic ring coupling of reserpine with 4-carboxyl-2,6dinitrobenzene diazonium ion with the consequent formation of an azo adduct. Optimization of reaction conditions and validation were carried out and the method applied to assay of reserpine in tablets.

Result: Reserpine coupled readily with CDNBD and optimization of experimental conditions showed the reaction to be completed in $10 \mathrm{~min}$ at room temperature. A 1:1 drug to reagent stoichiometric ratio was obtained for the azo adduct formed. The adduct exhibited a bathochromic shift with respect to the drug and pronounced hyperchromic shift with respect to the reagent. Sample analyses were done using a colorimeter at $470 \mathrm{~nm}$. The assays were linear and reproducible over the concentration range of 2.25 $24 \mu \mathrm{g} / \mathrm{mL}$. The new method was successfully applied in the assay of reserpine in tablets with a performance similar to the official (USP) spectrophotometric method $(p>0.05)$. This method represents a profound improvement on the previously reported colorimetric method for reserpine.

Conclusion: The method developed is rapid and could find application in in-process quality control of reserpine.
\end{abstract}

KEYWORDS: Reserpine, colorimetry, 4-Caboxyl-2,6-dintrobenzene diazonium ion (CDNBD), diazo coupling

*Correspondence: +234 8058427072 olakunleid@yahoo.com 


\section{INTRODUCTION}

Reserpine is an alkaloid obtained from the roots of certain species of Rauwolfia (Apocyanaceae) mainly $R$. serpentina and $R$. vomitoria or by synthesis. The material obtained from natural sources may contain closely related alkaloids ${ }^{1}$. Descriptions of the medicinal use of the root of Rauwolfia serpentina (Benth.), a climbing shrub indigenous to India, are present in ancient Hindu Ayurvedic writings ${ }^{2}$. Reserpine has been used in the management of hypertension and in chronic psychoses such as schizophrenia. It has also been tried in the management of Raynaud's syndrome

Chemically, reserpine is $(3 \beta, 16 \beta, 17 \alpha, 18 \beta$,

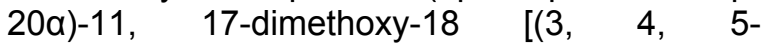
trimethoxybenzoyl)-oxyl] yohimban-16-carboxylic acid methyl ester or 3,4,5-trimethoxybenzoyl methyl reserpate ${ }^{3}$. Only the active pharmaceutical ingredient is official in the BP $2002{ }^{4}$ and determined by a UV procedure after nitrosation. The USP 24/NF $19^{5}$, uses a UV method for all Rauwolfia preparations after exhaustive and extensive solvent extraction. Other multi-ingredient preparations containing reserpine are assayed by HPLC methods in the USP 24/ NF 19. The determination of reserpine and other indole alkaloids from Rauwolfia serpentina and $R$. vomitoria by HPLC and HPTLC has been reported. Best separation on HPLC was achieved with $10 \% \mathrm{CH}_{3} \mathrm{CN}$ and $0.1 \%$ trifloroacetic acid in water $^{6}$. Qualitative and quantitative analyses of a reserpinechlorothiazide mixture in two steps by HPLC have also been described ${ }^{7}$ Other chromatographic procedures are also described $^{8}$.

Many flourimetric procedures have been described for reserpine either in bulk, dosage forms or in biological fluids. Agents that have been used include hydrogen peroxide, selenious acid, $p$-toluenesulphonic acid and vanadium pentoxide ${ }^{9}$, hexa-amine cobalt (III) tricarbanato cobaltate $^{10}$ and 2-iodoxybenzoate in aqueous acetic acid $^{11}$. A flow-injection assembly has also been adopted following fluorescence derivatization ${ }^{11}$.

A chemiluminometric determination of reserpine and two other Rauwolfia alkaloids, rescinnamine and yohimbine based on reaction with $\mathrm{KMnO}_{4} /$ polyphosphoric acid has been described $^{12}$.

Extraction of reserpine into chloroform from $\mathrm{pH}$ 4.0 phosphate buffer and then ion-pair formation with bromocresol purple or methyl orange have also been reported ${ }^{9}$.

The most commonly used colorimetric procedure for reserpine involved oxidation of the compound to 3,4-didehydroreserpine with sodium nitrite and measurement of the absorbance of oxidation product at about $390 \mathrm{~nm}$. Oxidation of reserpine with nitrite in acetic acid followed by extraction into chloroform has also been done. Reserpine has also been analysed colorimetrically by reaction with vanillin, aminopyrimidine, xanthydrol (50 - 500 $\mu$ g), phenylisocyanate, iodine and sodium glyoxalate . $^{9}$

Many of the above mentioned procedures suffer from the disadvantages of complexity of method, extensive solvent extraction and are time consuming. In continuation of our work on the development of relatively simple colorimetric methods for the assessment of organic compounds of pharmaceutical importance ${ }^{13-17}$, we report a new colorimetric method for the determination of reserpine in bulk and tablet dosage form using the newly developed 4carboxyl-2, 6-dinitrobenzene diazonium ion (CDNBD) ${ }^{18-20}$.

\section{EXPERIMENTAL}

\section{Chemicals and Reagents}

Brinerdine (Novartis Pharma SPA Torre Annunziata Italy for Novartis Pharma AG Basle, Switzerland), Regroton (Norvatis Pharma AG Levent-Istanbul), reserpine (CRS number 92808, Sandoz Pharma), ethanol, ethylacetate, glacial acetic acid, concentrated sulphuric acid, sodium nitrite (all analytical reagents from BDH, Poole, England), pre-coated thin layer chromatographic plates $\mathrm{GF}_{254}, 0.2 \mathrm{~mm}$ (Merck, Germany), 4amino-3,5-dinitrobenzoic acid (ADBA) synthesized in our laboratory.

Equipment:

UV/VIS spectrophotometer (Unicam Aurora, Helios Scan Software v 1.1, Pye Unicam, England), analytical balance H80 (Mettler, UK), 
ultrasonic bath (Langford Electronics, UK), vortex mixer (Griffins and George Ltd, UK), digital colorimeter, model 6051 (Jenway, U.K.)

\section{METHOD}

Preparation of stock solutions

An optimized process was used for the preparation of the CDNBD reagent solution using ADBA in concentrated sulfuric acid as previously reported ${ }^{20}$.

Reserpine stock solution was made by dissolving $6 \mathrm{mg}$ in $10 \mathrm{ml}$ of glacial acetic acid. This gave $0.6 \mathrm{mg} / \mathrm{mL}$ solution.

\section{Optimization studies}

Temperature and reaction time were optimized using the method of steepest ascent ${ }^{21}$. Aliquot of the reserpine stock solution $(100 \mu \mathrm{L})$ was added to the reagent solution $(500 \mu \mathrm{L})$ in a test tube and the reaction mixture was mixed in a vortex mixer for $10 \mathrm{sec}$ followed by incubation in turn at $30,50,60$ and $80^{\circ} \mathrm{C}$ for 5 and 20 mins. Each determination was carried out in duplicate. The reaction was terminated by addition of ice-cold water $(5 \mathrm{~mL})$ to the reaction mixture kept in icebath. The aqueous solution was extracted with ethylacetate $(5 \mathrm{~mL})$ as previously optimized ${ }^{13}$ and kept in a vial wrapped with aluminum foil. A blank reagent solution was similarly prepared but replacing reserpine stock solution with glacial acetic acid. The absorption spectrum of the reaction mixture extract was determined against the absorption of the blank reagent extract, using the UV/VIS spectrophotometer. The absorption wavelength, $470 \mathrm{~nm}$, where the difference in absorptivity between the adduct and the reagent was optimal was selected for sample determination on the colorimeter.

The optimal reaction time was determined by adding aliquot of reserpine stock solution (100 $\mu \mathrm{L})$ in turn to the reagent solution $(500 \mu \mathrm{L})$ in eight test tubes. Coupling reaction was carried out by incubation at $60^{\circ} \mathrm{C}$ for $0,2,5,10,20,25$ and 30 minutes. Ethylacetate extracts of the reaction mixture was prepared as usual after each reaction time and the absorbance was measured at $470 \mathrm{~nm}$ using the colorimeter. An optimum reaction time was then determined as the time corresponding to the maximal absorption of the samples. All determinations were done in duplicates.

\section{Stoichiometric ratio of drug-reagent adduct formation:}

Equimolar solutions $(0.918 \mathrm{mM})$ of the reagent and the drug stock solution were prepared using the procedure described above. Into seven different test tubes, $0,0.25,0.33,0.50,0.67$, 0.75 and $1.0 \mathrm{~mL}$ of the reagent solution was added respectively. Each tube was then made up to $1.0 \mathrm{~mL}$ with drug stock solution. Blank determinations were similarly carried out using glacial acetic acid in place of the drug stock solution. The mixtures were mixed on a vortex mixer for $10 \mathrm{sec}$ and kept at $30^{\circ} \mathrm{C}$ for 10 minutes and extracted into $5 \mathrm{~mL}$ ethylacetate. The absorbance was measured at $470 \mathrm{~nm}$ against the blank and the absorbance values obtained were plotted against the mole fraction of the reagent solution. Each determination was carried out in duplicate.

\section{Assay of dosage forms}

Weight uniformity test was carried out on the two brands of the tablet obtained. For Brinerdine ${ }^{(R)}$ tablets, an amount of powdered tablet equivalent to $0.4 \mathrm{mg}$ reserpine was weighed out and dissolved in $20 \mathrm{~mL}$ chloroform. After dissolution, the solution was clarified and $2 \mathrm{~mL}$ aliquots were dried in test tubes. The sample was reconstituted in $0.25 \mathrm{~mL}$ glacial acetic acid and then diazotized with $0.5 \mathrm{~mL}$ of CDNBD reagent solution. The sample was treated as before.

For Regroton ${ }^{\circledR}$, an amount equivalent to $0.5 \mathrm{mg}$ was dissolved in $25 \mathrm{~mL}$ chloroform and treated as Brinerdine ${ }^{\circledR}$. The USP 2000 spectrophotometric procedure for reserpine and hydrochlorothiazide was adopted as reference procedure.

\section{Validation of methods}

Calibration lines using standard solutions of 0,3 , $6,12,18$ and $24 \mu \mathrm{g} / \mathrm{mL}$ reserpine were carried out using the optimal analytical conditions as described above. Linear regression analysis was used to calculate the slope, intercept and the coefficient of determination $\left(r^{2}\right)$ of each calibration line. The assay precision and accuracy were determined as documented by USP ${ }^{22}$. The limit of detection was computed as 
previously described as the analyte concentration giving a signal equal to the blank signal plus three standard deviations of the blank $^{23}$.

\section{Assessment of method selectivity}

Two approaches were adopted for the assessment of the selectivity of this new procedure for the assay of reserpine. In the first procedure, standard solutions of reserpine $(12 \mu \mathrm{g} / \mathrm{mL})$ were spiked into each of starch, gelatin, lactose, magnesium stearate and a mixture of these excipients. Sample analysis was done through the optimized procedure described for reserpine above. The accuracy was compared with that of reserpine reference standard solution. In the second approach, powdered samples of the two brands of tablets investigated and the reserpine reference sample were kept at $100^{\circ} \mathrm{C}$ in the oven for 5 hours. Afterwards, methanolic solution of the tablets and the reference were analysed for possible degradation products. Three separate chromatographic systems were adopted [EtOAc: $\mathrm{MeOH}$ (9:1); EtOAc: MeOH (6:4) and Hex: EtOAc $(5: 5)]$ in order to reveal the presence of any degradation products.

\section{Stability of the azo adduct under diffuse light} Standard test solutions containing $12 \mu \mathrm{g} / \mathrm{mL}$ of reserpine were prepared in four sample vials. Two of the vials were wrapped with aluminum foil, while the other two were left unwrapped. Both sets were kept on the laboratory bench. The absorbance reading of the extracts at $470 \mathrm{~nm}$ were taken at 30 minutes interval for a period of three hours.

\section{RESULTS}

Reserpine was found to couple instantly with CDNBD and the colour remained stable at room temperature for days. The adduct, however, lacked fluorescence often inherent in reserpine when the TLC plate (containing the adduct and pure sample of reserpine) was examined under the UV lamp at $365 \mathrm{~nm}$. This indicates the formation of a new compound.

The absorption of reserpine, the blank reagent and the azo adduct between them is presented in Figure 1. Reserpine was found to exhibit a major peak at $290 \mathrm{~nm}$ while there is no significant light absorption in the visible region. The spectrum of the adduct however revealed a new $\lambda_{\max }$ in the visible region at $420 \mathrm{~nm}$. The maximum difference in the absorptivity between the adduct and the reagent was found at $470 \mathrm{~nm}$. One peculiar feature of the absorption spectrum of the adduct is that it appears more of a hyperchromic shift when compared with that of the reagent $\left(\lambda_{\max } 255,340\right.$ and $\left.430 \mathrm{~nm}\right)$. However, the colour of the adduct (reddishbrown to bright orange) is distinct from that of the yellow colour of the reagent. Absorbance of the adduct was highest at $30^{\circ} \mathrm{C}$ and $10 \mathrm{~min}$ reaction time. Maximum absorbance of the adduct was obtained at a mole fraction of 0.5 for the reagent solution and the absorbance was found to decrease at both lower and higher mole fractions. The azo adduct formed by the coupling reaction was stable when wrapped for a period of 3 hours. There was no significant difference between the content of reserpine in the tablets assayed by the new method and the USP method as shown in Table 1 ( $p>0.05)$. The linear regression equation for the calibration line under the optimal conditions is $y=0.007 x+0.1012$ with a correlation coefficient of $0.9974\left(\mathrm{r}^{2}=\right.$ 0.9948). The limit of detection for the assay of reserpine using CDNBD was found to be $2.245 \mu \mathrm{g} / \mathrm{mL}$. The $95 \%$ confidence limit for the slope and intercept are $0.007 \pm 0.001$ and $0.1012 \pm 0.0091$ respectively. Recovery studies for the assay of reserpine using the CDNBD method were done at concentration levels of 6 , 12 , and $18 \mu \mathrm{g} / \mathrm{ml}$. The three-day assessment of the accuracy and reproducibility is presented in Table 2. The overall accuracy was found to be $101.17 \pm 3.16$ with a coefficient of variation of $3.12 \%$. The photostability of the reserpine azo adduct to diffuse light in ethylacetate done over three-hour period revealed that for samples exposed to the laboratory atmosphere, the absorbance was found to drop gradually to a constant value of 0.16 from 90 to 180 minutes. The absorbance of the wrapped samples was however constant over the three-hour period.

In the assessment of method selectivity, the following recoveries were obtained in the presence of the excipients; starch (102.39 \pm 
Idowu et al

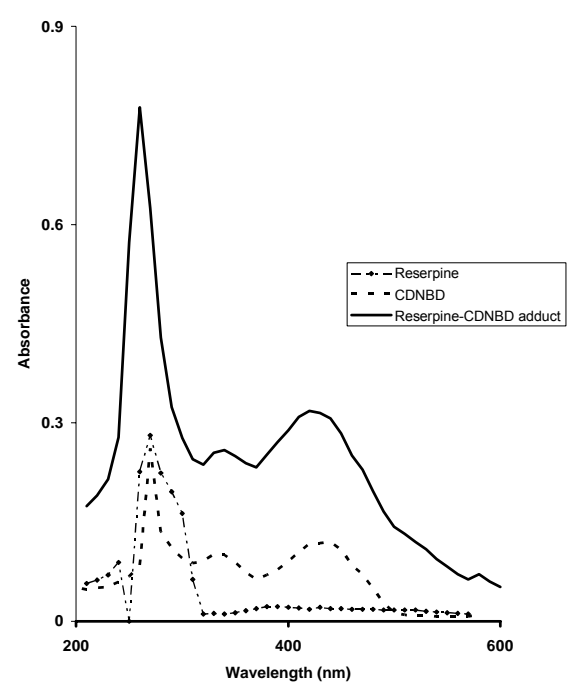

Overlaid absorption spectra of Reserpine, CDNBD and Reserpine-CDNBD azo adduct

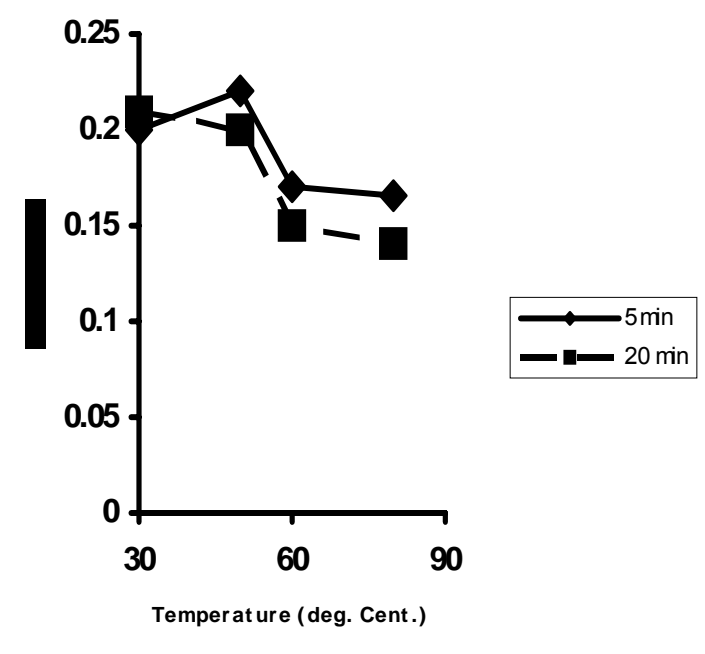

(2) Optimization of coupling reaction temperature at two time levels

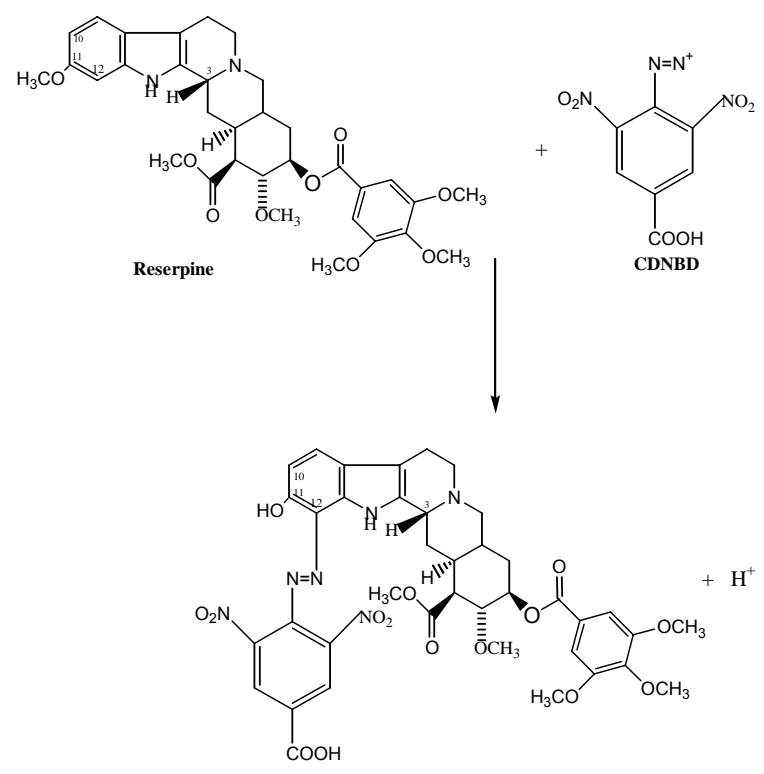

(3) Coupling reaction between CDNBD and reserpine 
2.51), lactose (103.40 \pm 3.1$)$, magnesium stearate $(103.49 \pm 2.90)$, gelatin $(102.69 \pm 2.49)$ and a mixture of these excipients $(102.39 \pm 2.51)$ when compared with the reserpine alone $(103.9 \pm 3.0)$. In the second approach, the tablet samples gave a single spot in the three mobile phases investigated. It appears that reserpine is thermally stable.

\section{DISCUSSION}

The colorimetric determination of reserpine via diazo coupling reaction, using CDNBD, is the first of such methods to be carried out. While most previously reported methods are based on measurement of the fluorescence of reserpine, a cheaper and readily adaptable colorimetric method would be desirable.

Optimization of coupling reaction temperature was investigated at $30,50,60$ and $80^{\circ} \mathrm{C}$ at both 5 and 20 minutes. The optimization of reaction temperature is depicted in Figure 2. One prominent observation is that at 5 and 20 minutes, the absorbance of the adduct gradually falls after $50^{\circ} \mathrm{C}$. At 5 minutes, a very slight increase (0.02 absorbance units) was observed when the temperature was increased by $20^{\circ} \mathrm{C}$ (i.e. $30^{\circ} \mathrm{C}$ to $50^{\circ} \mathrm{C}$ ). The absorbance of the azo adduct thereafter dropped. Similarly, the absorbance at $30^{\circ} \mathrm{C}, 20$ minutes is only 0.01 higher than that at $30^{\circ} \mathrm{C}, 5$ minutes. Since a gain in time of analysis will be achieved on using the most convenient temperature, the room temperature of $30^{\circ} \mathrm{C}$ was selected as the temperature for the reaction of reserpine with CDNBD. The optimization of coupling time gave 10 minutes as the optimum time.

Maximum absorbance for the reserpine azo adduct was observed at the mole fraction of reagent being 0.5 . This implies that the reserpine molecule required just a molecule of CDNBD for the dye formation. The absorbance gradually increased from lower mole fractions of the reagent and peaked at 0.5 ; the value decreased thereafter. The ${ }^{1} \mathrm{H}$ NMR of reserpine reveals that the proton on the indole ortho $(\mathrm{C}-12)$ to the methoxyl group is the most shielded ${ }^{24}$. Thus electrophilic attack by CDNBD will be on this position. Judging from the spectra observed for indomethacin $^{16}$ which shares the indole nucleus with reserpine, the structure of reserpine-
CDNBD adduct is proposed as shown in Figure 3 . This is further corroborated by the $1: 1$ mole ratio obtained between reserpine and CDNBD.

The recovery studies showed results that represent a great improvement over other previously reported spectrophotometric methods that adopted non-specific reactions for isolation and determination of reserpine. This high recovery obtained here is comparable with those obtained with some flourimetric methods especially that described by Walash et al ${ }^{10}$ where the recovery of $100.8 \pm 1.4 \%$ was obtained.

The lower concentration value adopted (6 $\mu \mathrm{g} / \mathrm{mL}$ ) was found to give the least precise results. This can be attributed to the fact that it is close to the limit of quantitation (LOQ $=3 \times$ LOD $=6.7335 \mu \mathrm{g} / \mathrm{mL}$ ). However, better recoveries were observed for 12 and $18 \mu \mathrm{g} / \mathrm{ml}$ analyte sizes. Since $12 \mu \mathrm{g} / \mathrm{mL}$ represents the midpoint of the calibration line and since it also gave precision results close to $18 \mu \mathrm{g} / \mathrm{mL}$ concentration levels, it was selected as working concentration for subsequent analysis.

The result of the photostability study revealed that the new method (CDNBD) has a common property with all methods (including the BP and USP methods) used for the analysis of reserpine and that, all sample solutions be protected from light. However, if the analysis could be carried out within thirty minutes in the CDNBD method only 0.03 absorbance loss will be observed (from 0.21 at 0 minute to 0.18 at 30 minutes). It can therefore be concluded that the azo adduct of reserpine is stable up to three hours when sample solutions in ethylacetate are wrapped in aluminum foil.

This new procedure described for reserpine for the first time offers a great advantage of simplicity. It is also the first colorimetric procedure for reserpine based on azo dye formation. We have previously demonstrated the reactivity of CDNBD as a useful agent for the detection and assay of phenol ethers 16, 17, 25. Essentially, the ability of the method to determine reserpine in multicomponent formulations without recourse to extensive extraction procedure are of great advantage. 


\section{Idowu et al}

Table 1: Assay of reserpine in Brinerdin ${ }^{\circledR}$ and Regroton ${ }^{\circledR}$

\begin{tabular}{lcccc}
\hline Tablet brand & CDNBD method $^{\mathrm{a}}$ & USP method & \multicolumn{2}{c}{$\mathrm{p}$-value } \\
& $104.3 \pm 1.5$ & $101.2 \pm 1.7$ & 0.32 & 0.07 \\
\hline Brinerdin $^{\circledR}$ & & & & \\
& & & & \\
& $102.8 \pm 2.5$ & $101.5 \pm 1.4$ & 0.18 & 0.37 \\
Regroton $^{\circledR}$ & & & & \\
\hline
\end{tabular}

USP requirement: content of reserpine $=90-110 \%$ of labeled amount a; $n=5 \quad b ; n=4$

Table 2: Assessment of accuracy and repeatability of the new method of assay of reserpine

\begin{tabular}{|c|c|c|c|c|c|c|}
\hline \multirow{2}{*}{$\begin{array}{l}\text { Concentration } \\
\qquad(\mathrm{g} / \mathrm{mL})\end{array}$} & \multicolumn{2}{|c|}{ Day 1} & \multicolumn{2}{|c|}{ Day 2} & \multicolumn{2}{|c|}{ Day 3} \\
\hline & Mean \pm S.D* & RSD\% & $\begin{array}{l}\text { Mean } \pm \\
\text { RSD\% }\end{array}$ & S.D* & Mean \pm S.D* & RSD \% \\
\hline 6 & $95.0 \pm 6.0$ & 6.3 & $100.9 \pm 6.0$ & 5.9 & $98.0 \pm 6.9$ & 7.0 \\
\hline 12 & $103.9 \pm 3.0$ & 2.9 & $102.4 \pm 3.4$ & 3.4 & $103.9 \pm 3.0$ & 2.9 \\
\hline 18 & $104.8 \pm 3.8$ & 3.6 & $101.8 \pm 0$ & 0 & $99.9 \pm 2.3$ & 2.3 \\
\hline
\end{tabular}

Between-day statistics $=101.2 \pm 3.2 \%$ (Mean \pm s.e.m), RSD (of s.e.m) $=3.1 \%$

${ }^{*} n=9$, Regression equation: $y=0.007 x+0.1012\left(r^{2}=0.9948\right)$ 
This new method via azo dye formation gave higher accuracy than the chemiluminometric determination of Pinotsis et al ${ }^{12}$. When compared with the use of vanillin and xanthydrol as derivatizing reagents, CDNBD method is more sensitive. Another advantage, when compared with the reference USP method, is that this method offers some degree of selectivity. The USP method uses nitrous acid generated in situ for nitrosation and any residual or contaminant aromatic skeleton will also react. However, CDNBD will only react with an activated skeleton.

\section{CONCLUSION}

This new method for reserpine represents an improved approach for its determination. It offers the advantages of speed, simplicity and selectivity. Moreover, colorimeters are readily available and affordable. It is of equivalent accuracy with the official procedure and it could find application as an in-process analytical method for reserpine. The utilization of CDNBD as a pre-column derivatization reagent for HPLC analysis of reserpine is under investigation in our laboratory.

\section{REFERENCES}

1. Partiff $\mathrm{K}$ Martindale: The Complete drug reference Ed 32. The Pharmaceutical Press, London, 1999, p. 942.

2. Oates JA and Brown NJ. Antihypertensive agents and the drug therapy of hypertension In: Hardman JG and Limbird LE (eds). Goodman and Gilman's The pharmacological basis of Therapeutics Ed 10. McGraw-Hill Medical Publishing Division N.Y., 2001, pp. 882-884.

3. Budavari $S$ (ed.). Merck Index: An Encyclopedia of Chemicals, Drugs and Biologicals. Ed 12. Merck Research laboratories, Division of Merck and Co. Inc. N.J., 1996, 1400-1401.

4. British Pharmacopoeia. The Pharmaceutical Press, London, 2002; p. 1487

5. United states Pharmacopoeia USP 24 /NF 19. United States Pharmacopoeial Convention Inc. Rockville, USA, 2000, pp. 1466-1468, 1470-1478

6. Klyushnichenko VE, Yakimov SA, Tuzova TP, Syagailo Ya V, Kuzovkina IN, Wulfson AN, Miroshnikov Al. Determination of indole alkaloids from $R$. serpentina and $R$. vomitoria by highperformance liquid chromatography and highperformance thin-layer chromatography. J. Chromatogr. A 1995; 704: 357-362.

7. Honigberg IL, Stewart JT, Smith AP, Plunkett RD and Hester DW Liquid chromatography in pharmaceutical analysis II: determination of a reserpine-chlorothiazide mixture. J. Pharm. Sci. 1974; 63: 1762-1764.

8. Muhtadi FJ. Reserpine. Analytical profiles of drug substances. Florey K (ed.). Academic Press, Inc, USA. 1984; 13: 749-753.

9. Schirmer RE. Reserpine. Analytical profiles of drug substances. Florey K (Ed.). Academic Press Inc USA. 1975; 4: 384-430.

10. Walash MI, Belal F, Aly FA. Flourimetric determination of reserpine in dosage forms. Talanta 1988; 35: 731-733.

11. Calatayud JM, Benito CG. Photochemical derivatization and fluorimetric determination of reserpine in a flow-injection assembly. Anal. Chim. Acta. 1991; 245: 101-107.

12. Pinotsis N, Calokerinos AC, Baeyens WRG Chemiluminometric determination of reserpine and related alkaloids. Analyst 2000; 125: 1307-1311.

13. Idowu SO, Tambo SC, Adegoke AO, Olaniyi AA Novel colorimetric assay of mefenamic acid using 4-amino-3,5-dinitrobenzoic acid (ADBA).Trop J Pharm Res 2002; 1:15-22.

14. Idowu SO, Adegoke OA and Olaniyi AA (2004): Colorimetric Assay of propranolol by derivatization: Novel application of diazotized 4-amino-3, 5dinitrobenzoic acid (ADBA), J. AOAC Intern. 2004; 87: $573-578$

15. Idowu SO, Adegoke OA, Oderinu BA and Olaniyi AA Rapid colorimetric assay of diclofenac sodium tablets using 4-carboxyl-2,6-dinitrobenzene diazonium ion (CDNBD). Pak J Pharm Sci 2006; $19: 134-141$

16. Adegoke OA, Idowu SO, Olaniyi AA. Novel Colorimetric determination of indomethacin using 4-carboxyl-2,6-dinitrobenzene diazonium ion. Acta Pharmaceutica,2006; 56 : 189-202

17. Adegoke OA, Idowu SO, Olaniyi AA. A new spectrophotometric method for determination of nadolol. J Ir Chem Soc 2006; 3: 277-284

18. Idowu SO. Ph.D. Thesis 1998; University of Ibadan, Nigeria

19. Idowu SO, Olaniyi AA. Evaluation of diazotized 4amino-3,5-dinitrobenzoic acid (ADBA) as a new derivatizing reagent. Afr. J. Med \& Med. Sci. 2001 30: $217-220$

20. Idowu SO, Kolawole AO, Adegoke OA, Kolade YT Fasanmade AA \& Olaniyi AA. Kinetics of thermal decomposition of 4-Carboxyl-2,6dinitrobenzenediazonium ion (CDNBD), J.AOAC Int. 2005; 88:1108-1113

21. Miller JC, Miller JN. In Statistics for Analytical Chemistry Ed 3, Prentice Hall, London, Ellis Horwood, PTR, 1993, pp.188-190

22. United states Pharmacopoeia USP 24 /NF 19. United States Pharmacopoeial Convention Inc. Rockville, USA, 2000, p. 2149

23. Miller JC, Miller JN. In Statistics for Analytical Chemistry $3^{\text {rd }}$ Ed., Prentice Hall, London, Ellis Horwood, PTR, 1993, pp. 103-104, 188-190

24. Mills III T, Roberson C, McCurdy $\mathrm{HH}$, Wall WH. Instrumental Data for Drug Analysis Ed 2. CRC Press, Boca Raton, USA, 1993, p. 2037 


\section{Idowu et al}

25. Adegoke OA, Idowu SO, Lawal MO, Olaniyi AA (2005): 4-Carboxyl-2,6-Dinitrobenzene Diazonium ion : A new Diazonium for detection of phenol ether homologues. J. Pharm. \& Bioresources, 2005; 2 :146-161 